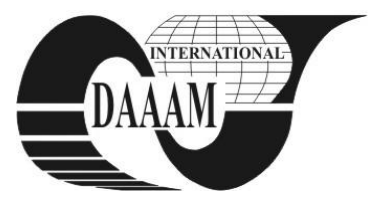

Annals of DAAAM for 2011 \& Proceedings of the 22nd International DAAAM Symposium, Volume 22, No. 1, ISSN 1726-9679 ISBN 978-3-901509-83-4, Editor B. Katalinic, Published by DAAAM International, Vienna, Austria, EU, 2011 Make Harmony between Technology and Nature, and Your Mind will Fly Free as a Bird

Annals \& Proceedings of DAAAM International 2011

\title{
AUTOMATED WORKSPACE-VISUALIZATION FOR SERIAL AND PARALLEL ROBOTS
}

\author{
LIEBE, A[lexander] R[ichard] \& HIEGER, C[hristof]
}

\begin{abstract}
In this article a numerical MATLAB algorithm is presented to visualize the workspace of serial and parallel kinematics. For serial kinematics the Monte Carlo Method is used to convert the inputted DH-Parameters to a scatter plot of the reachable workspace. The article gives also a detailed insight into the processing of these scatter plot to find and visualize the boundary points of the workspace. With these boundary points the algorithm generates sectional views in all three dimensions and a three-dimensional visualization. For parallel kinematics the algorithm can import a given scatter plot from an external MAT-file. The result of the algorithm is shown on a serial kinematic with six degrees of freedom $(A B B$ IRB 140).
\end{abstract}

Key words: workspace, visualization, robot, Monte Carlo method

\section{PROBLEM STATEMENT}

As they need to interact with their work environments, the reachable workspace of industrial robots is a very important criterion for their implementation. The calculation and visualization of the robot workspace is a necessary and powerful tool to support and accelerate the selection procedure. Contrary to previous algorithms, which need specific robotic and mathematical knowledge for their understanding, the aim of this research is to design a numerical algorithm using the softwaretool MATLAB to determine and visualize the workspace. The technical expertise of these visualizations can be used to enhance the designing process of industrial robots.

\section{EXISTING METHODS AND APPROACHES}

The first research in workspace-visualization was done by Gupta (1986). He linked the kinematical characteristics of an industrial robot to its workspace and presented an algorithm to determine the planar boundary. In the following years two essential methods arose.

The analytical method uses complex mathematical operations to transform the kinematic equations into usable sets of surfaces, which can then be visualized. This approach was promoted by Abdel-Malek \& Yeh (1997). It is also possible to calculate the workspace from the Jacobian-matrix, with a singularity-analysis. This complex, mathematical algorithm was developed by Goyal \& Sethi (2010). There are also iterative approaches (Gupta et al., 1983) and approaches using Fouriertransformation to determine the workspace of an industrial robot.

On the other hand there were attempts to simplify the visualization with numerical evaluations (Kumar \& Patel, 1986). Besides the approach with inverse kinematics, the approximated workspace can be quantized in discrete planes. Within this dataset an alghorithm searches for the boundarypoints, which can be identified and visualized for the user. To calculate the approximate workspace of an industrial robot the Monte Carlo Method was used by Alciatore \& Chung-Ching (1994).

\section{REALIZATION AND RESULTS}

\subsection{The Graphical User Interface (GUI)}

A GUI has been designed to simplify the manipulator process for the user. To process serial kinematics the user can enter the DH-parameters $\left(a_{i}, \alpha_{i}, d_{i}\right.$ and $\left.\theta_{i}\right)$ into a template. After entering the parameters, the algorithm calculates the entire transformation-matrix of the robot by multiplying the single transformation-matrices together.

The interface provides also an import file to visualize a given scatter plot from a MAT-file. With this aid the user is able to visualize the workspace of a parallel robot.

\subsection{Determining the Workspace}

The Monte Carlo Method provides a simple and efficient approach to calculate an approximate scatter plot from the entered DH-table of a serial robot. The algorithm allows the processing of serial kinematics with linear and rotatory joints. The complete method is shown in Fig. 1.

To determine the workspace of a parallel robot the algorithm allows the user to import a scatter plot from a MATfile. On that account the Monte Carlo Method is skipped and the imported scatter plot is visualized.

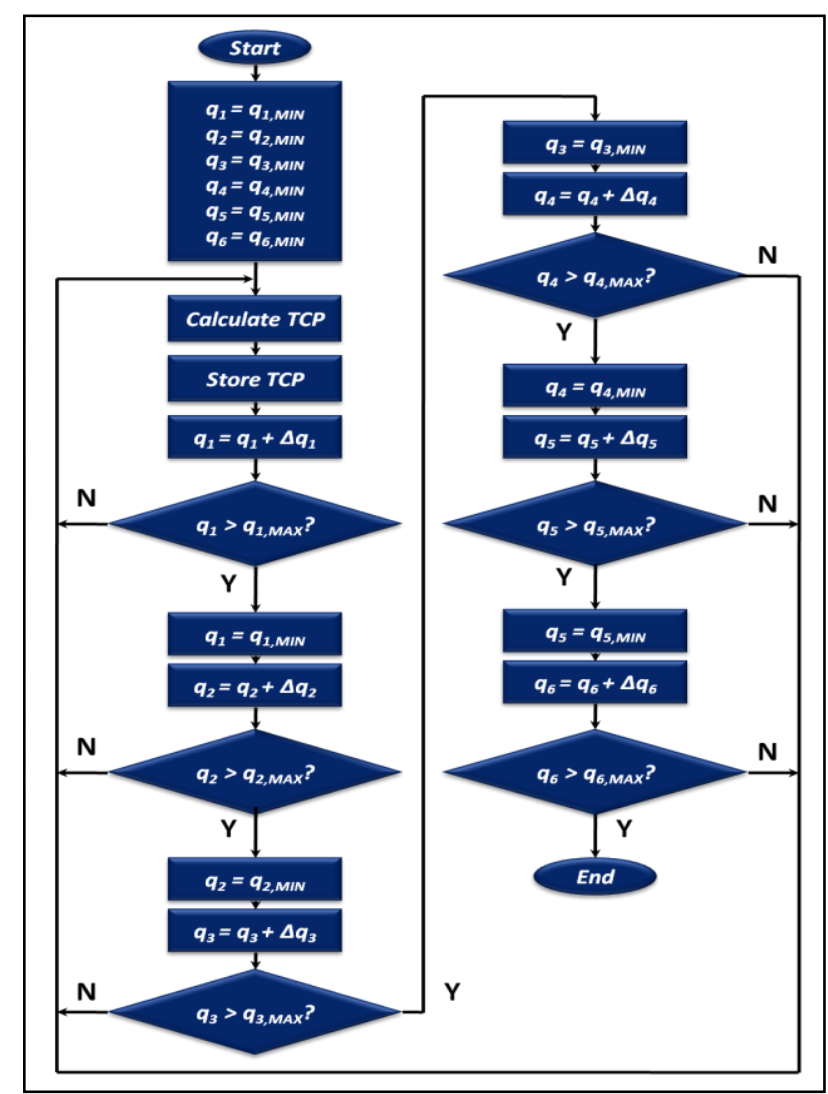

Fig. 1. Monte Carlo Method for a $6 \mathrm{DoF}$ 


\subsection{D-Visualization}

After receiving the scatter plot, the workspace-area is defined and quantized in $\mathrm{x}-, \mathrm{y}-$, and $\mathrm{z}$-segments. Within all three planes (xy-, yz- and xz-plane) the algorithm scans each segment for local minima and maxima. These points are then linked together and formed into the two-dimensional plot as shown in Fig. 2 as an example.

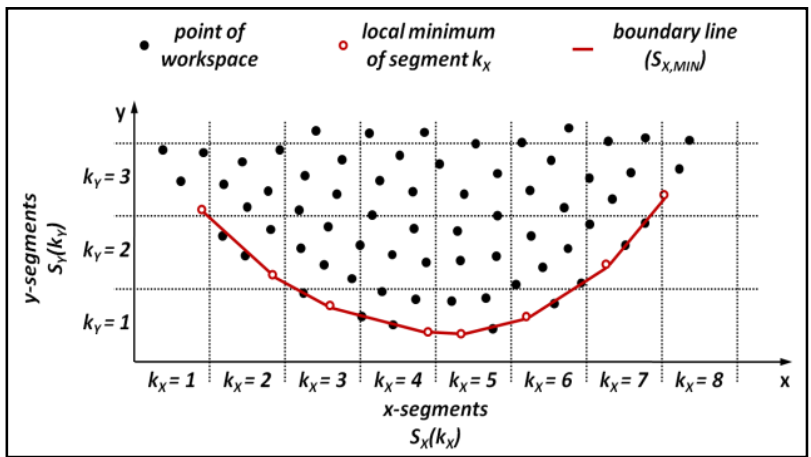

Fig. 2. Minima of the xy-plane

This procedure is done for all three planes. The final boundaries of all three two-dimensional plots are produced by combining the minima and maxima in the correct order (1-3).

$$
\begin{aligned}
& B_{X Y}=S_{X, M I N} \cup S_{X, M A X} \cup S_{Y, M I N} \cup S_{Y, M A X} \\
& B_{Y Z}=S_{Y, M I N} \cup S_{Y, M A X} \cup S_{Z, M I N} \cup S_{Z, M A X} \\
& B_{X Z}=S_{X, M I N} \cup S_{X, M A X} \cup S_{Z, M I N} \cup S_{Z, M A X}
\end{aligned}
$$

In addition to the planar boundaries the algorithm calculates and shows the relevant overall dimensions of the workspace (Fig. 3).

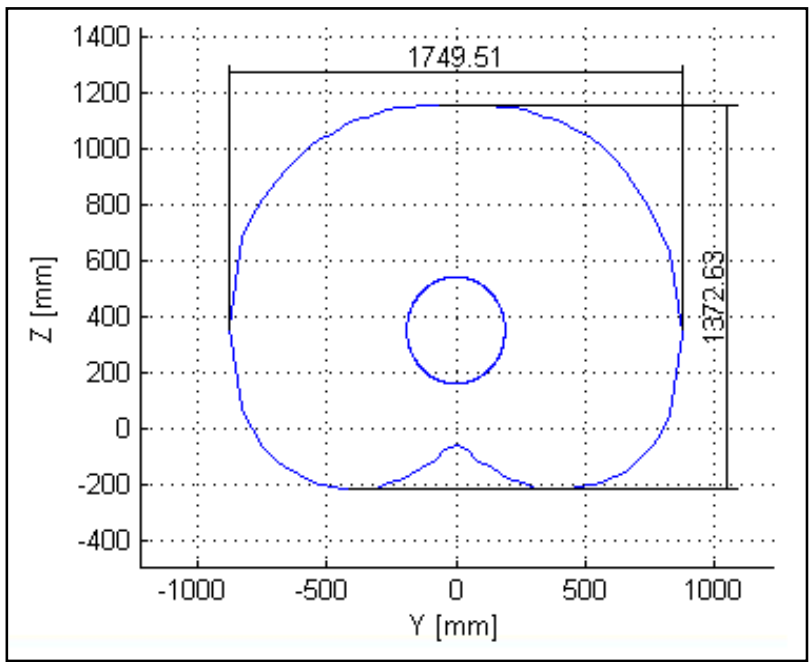

Fig. 3. Final 2D-visualization of ABB IRB $140\left(B_{\mathrm{XY}}\right)$

\subsection{D-Visualization}

The three-dimensional visualization is similar to the twodimensional. The algorithm combines the segment-dimensions $\left(S_{X}, S_{Y}\right.$ and $\left.S_{Z}\right)$ to planar segment-matrices $\left(S_{X Y}, S_{Y Z}\right.$ and $\left.S_{X Z}\right)$. The cells of these segment-matrices are then scanned in the perpendicular dimension for local minima and maxima. These two data sets represent two surfaces, which are combined together (4).

$$
B_{3 D}=S_{X Y, M I N} \cup S_{X Y, M A X}
$$

To merge these surfaces the edges are determined and set to the same level. The final surfaces are then plotted to the threedimensional plot (Fig. 4).

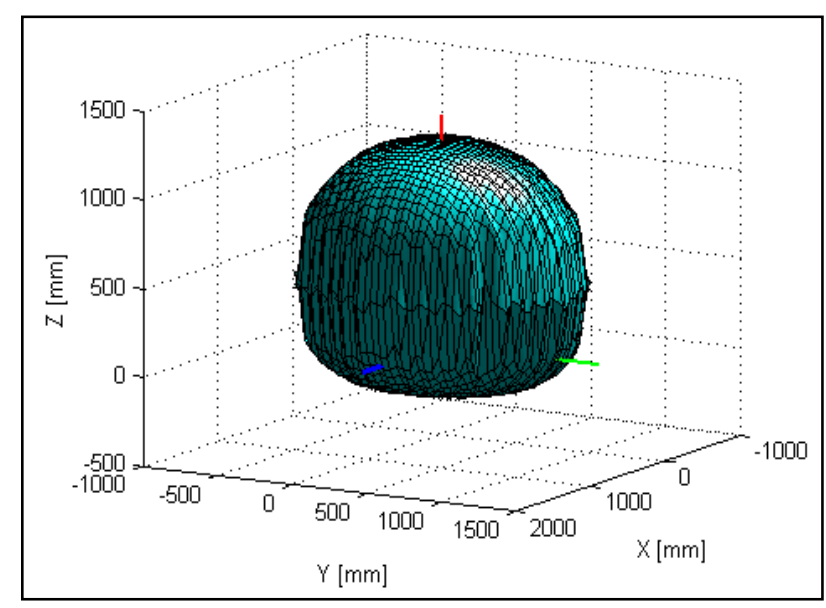

Fig. 4. Final 3D-visualization of ABB IRB $140\left(B_{3 D}\right)$

\section{CONCLUSION AND FURTHER RESEARCH}

A numerical algorithm to determine and visualize the workspace of serial or parallel robots is presented. The implemented MATLAB-algorithm computes a scatter plot of the workspace using a simple method, called the Monte Carlo Method.

This scatter plot is then quantized into $\mathrm{x}-, \mathrm{y}-$ and $\mathrm{z}-$ segments. Within these segments local minima and maxima are searched out. The minima- and maxima-points of each dimension are then linked together and printed as three twodimensional plots. For the 3D-visualization the segmentdimensions are combined to segment-matrices. Within these matrices local minima and maxima are searched out, linked together and printed to the desired plot. The results have been demonstrated on an ABB IRB 140.

Further research could be done, by integrating the algorithm into a CAD system. The algorithm would then be able import the kinematical characteristics from the CAD robot-model to determine and visualize the workspace. Depending on the CAD data the algorithm can also consider the inner collision-zone of the robot and modulate the visualizations. The integration of the algorithm into a CAD system would ease further work in robotdesign and collision-avoidence for industrial application.

\section{REFERENCES}

Abdel-Malek, K. \& Yeh, H.-J. (1997). Analytical Boundary of the Workspace for General 3-DoF Mechanisms, The Journal of Robotics Research, Vol. 16, No. 2, (April 1997) 198-213, ISSN 0278-3649

Alciatore, D. \& Chung-Ching, N. (1994). Determining Manipulator Workspace Boundaries using the Monte Carlo Method and Least Square Segmentation, Available from: http://www.engr.colostate.edu/ dga/dga/papers/workspace. pdf Access: 2011-03-09

Goyal, K. \& Sethi, D. (2010). An analytical Method to find Workspace of a Robotic Manipulator, Journal of Mechanical Engineers, Vol. 41, No. 1, (June 2010) 25-30, ISSN 0379-4318

Gupta, K. (1986). On the Nature of Robot Workspace, The International Journal of Robotics Research, Vol. 5, No. 2, (Summer 1986) 112-121, ISSN 0278-3649

Gupta, K.; Hansen, J. \& Kazerounian, S. (1983). Generation and Evaluation of the Workspace of a Manipulator, The International Journal of Robotics Research, Vol. 2, No. 3, (Fall 1983) 22-31, ISSN 0278-3649

Kumar, A. \& Patel, M. (1986). Mapping the Manipulator Workspace using Interactive Computer Graphics, The Journal of Robotics Research, Vol. 5, No. 2, (Summer 1986) 122-130, ISSN 0278-3649 